\title{
Random Forest Regression Based on Partial Least Squares
}

\author{
Connect Partial Least Squares and Random Forest
}

\author{
Zhulin $\mathrm{Hao}^{1}$, Jianqiang $\mathrm{Du}^{1 *}$, Bin $\mathrm{Nie}^{1}$, Fang $\mathrm{Yu}^{1}$, Riyue $\mathrm{Yu}^{2}$ and Wangping Xiong ${ }^{1}$ \\ ${ }^{1}$ Computer School, Jiangxi University of Traditional Chinese Medicine, Nanchang, 330004, China \\ ${ }^{2}$ College of Pharmacy, Jiangxi University of Traditional Chinese Medicine, Nanchang, 330004, China \\ *Corresponding author
}

\begin{abstract}
Partial Least Squares (PLS) Regression is lack of theoretical guidance of rules to achieve the nonlinear by the quasi linearization rule, and its accuracy declines in the face of the unknown variables distribution. Furthermore, the loss of information is easy to arise for the mean processing of the leaf in the Regression Tree of the traditional Random Forest Regression. On this basis, Partial Model Tree (PMT) is proposed combining Partial Least Squares Regression with Regression Tree, to achieve the nonlinear regression by constructing multiple linear fragments of Partial Least Squares to complete linear approximation of the unknown variables, and the information loss issue caused by that the leaf nodes are treated by direct mean processing is avoided, when PLS regression is used in the leaf nodes. It applies PMT to ensemble learning to build Partial Least Squares of Random Forests Regression (PLS-RFR), improving the generalization ability of PMT. The ability of explanation and predicting get improved in the experiment data of MaXingShiGan decoction of the monarch drug to treat the asthma or cough and five sample sets in the UCI Machine Learning Repository. Finally, it verifies that the PMT and RFPLS possess a certain degree of validity and correctness.
\end{abstract}

Keywords-partial least square; regression tree; random forest; linear approximation; TCM information

\section{INTRODUCTION}

The traditional Random Forest Regression (RFR) is a way of calculating the final predicted value. The procedure are as follows: firstly, using Bootstrap [1] to get the random sampling and extracting the training dataset in the original sample dataset; secondly, constructing various copies of the training dataset of distinct size; thirdly, creating distinct versions of the single basic regression tree in the every replica training dataset and calculating average value through the predicted value in all basic regression trees. But the basic regression tree, a kind of poor single learning machine, using the average value method to handle the leaf nodes directly, makes the system lose some valuable information when a leaf node has a suitable sample. Apparently, when needs to integrate of multiple versions of regression trees, the traditional Random Forest Regression has poor ability to explain the model. Meantime, the pruned regression tree is also very tedious and hard to explain, although regression tree provides the pruning method in the leaf node for the information loss. Basing on this, in the process of handling the leaf nodes, Quinlan [2] has provided a linear regression equation algorithm, which replace the average processing method in traditional regression tree, namely, M5 Model Tree. The Model Tree sets up a piecewise linear function by the leaf node, and divides the complex nonlinear system information into several multiple linear segments, has a piecewise linear approximation to any unknown variable distribution trend. Haijun $\mathrm{Li}$ et al [3] have proposed a mixed learning regression algorithm utilizing Naive Bayes in the leaf node of the Regression Tree, and the algorithm achieves good results in the UCI machine learning. Using LogitBoost to build Overlay regression in the leaf node of the decision tree, Kun Zhang et al [4] get a new LC Tree Model Tree algorithm which can analyze the feature of telecom users to predict the number of people offline. Besides, Partial Least Squares regression (PLSR) [5] solves the problem that traditional Multiple Regression can still be effective to construct regression model when the Gauss Markov assumptions invalidate. There are no strict restriction for the capacity of sample and the severity of independent variables multicollinearity and the variable number. Huiwen Wang et al [6] have detailedly discussed the application of Partial Least Squares in the multicollinearity elimination and auxiliary analysis and quasi-linearization aspects, verifying that Partial Least Squares has great benefits in system information recognition and modeling reliability comparing to traditional multiply regression. But Partial Least Squares nonlinear method is a quasi-linearization regression method that conducts the nonlinear preprocessing transformation of the data using quasi linear rules [7]. The transformation can't reflect the nonlinear features of the data when facing the unknown knowledge, and lack of theoretical guidance. At this moment, Partial Least Squares nonlinear model is not well in precision. Considering the insufficient of Partial Least Squares method in the nonlinear and the deficiency of the M5 model tree needing to satisfy Gauss Markov assumptions when using classical multiple regression to deal with leaf nodes, it adopts Partial Least Squares to process the leaf node of the M5 Model Tree. According to the model, Random Forest Regression of Partial Least Squares is constructed by multiply distinct versions of this modified M5 model. This method, achieving the nonlinear regression by constructing multiple linear fragments of Partial Least Squares to complete linear approximation for the unknown variables, making information loss issue gets avoided when the leaf nodes is directly for mean with appropriate samples, optimizes 
the traditional Random Forest Regression and makes up the flaw of Partial Least Squares method in the nonlinear.

\section{Partial Least Squares Model Tree}

The Model Tree is obviously far superior to the conventional linear regression or the basic regression tree, adopting multiple linear regression model to nonlinear approximation of a continuous function. Not only is the Model Tree smaller and easier to be explained than the basic regression tree, but also much lower error generated in the sample data. The traditional Model Tree is built by mending the basic regression tree with using two important steps about pruning and smoothing, remedying the fault of the multiply regression. However, it can't be able to be integrated in the ensemble learning. One of necessary conditions for ensemble learning is a weak learner and the pruning significantly increases the computational time and space. Therefore, Partial Model Tree (PMT) is proposed, adopting Partial Least Squares regression to improve the traditional Model Tree.

Definition 1: Dividing the sample data into two parts by the principle of maximum change of variance, there are some conditions to determine whether it continues dividing or not, one of the conditions is the maximum allowable value of decreased space of leaf, the other is maximum allowable number of sample of leaf, if the node is the leaf then Partial Least Squares or the average method is handled according to the basic information of the sample. The regression tree is called as Partial Model Tree (PMT).

PMT doesn't construct a basic regression tree directly, after dividing the tree in a rule of the maximum fluctuation of total variance decline space, it selects average method or PLSR to build the linear model as leaf node directly. PLSR is especially suitable to solve the sample data where there is a feature of small sample size, multicollinearity and existing system noise. Therefore, PMT is better in those aspects than the basic regression tree and the traditional Model Tree for the same sample data. Similarly, it is easier for the pruning of PMT and increases the accuracy by the smoothing of Model Tree. The main algorithm in the PMT is as follows:

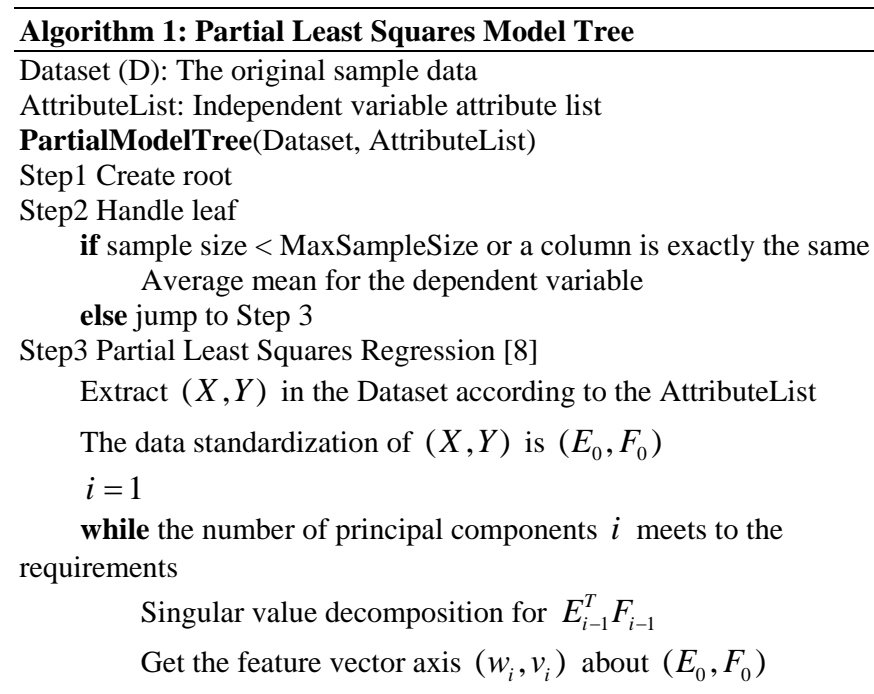

$$
\text { Calculate principal component score } t_{i}=E_{i-1} w_{i} \text { and }
$$$$
u_{i}=F_{i-1} v_{i}
$$$$
\text { Loading vector is } p_{i}=X_{i-1}^{T} t_{i} /\left\|t_{i}\right\|^{2} \text { and } r_{i}=F_{i-1}^{T} t_{i} /\left\|t_{i}\right\|^{2}
$$$$
\text { Regression equation is } E_{i-1}=t_{i} p_{i}^{T}+E_{i} \text { and }
$$

$F_{i-1}=t_{i} r_{i}^{T}+F_{i}$

$$
\text { Residual information matrix is } E_{i} \text { and } F_{i}
$$

end

Integrate Partial Least Squares equation

Step 4 Dividable attribute judgment

Divide sample set according to each attribute of the AttributeList Each attribute is divided into a sample set

Calculate the dividable mixed total variance after dividing the sample set

Calculate the mixed total variance before dividing the sample set Step 5 Dividable judgment

if the total variance after partition - the total variance before partition > MaxDrop

Divide the sample data by (bestAttribute,

bestAttributeValue)

Get the sample subset $\mathrm{T} 1$ and $\mathrm{T} 2$ after partition

else jump to Step 3

Step 6 Recursion

Recursion in the sample subset T1 and construct Partial Model Tree on (T1, AttributeList)

Recursion in the sample subset T2 and construct Partial Model

Tree on (T2, AttributeList)

Step 7 end

The Algorithm 1 mainly involves two important parameters. First is the maximum allowable number of sample (MaxSampleSize) in the leaf nodes. It is usually fixed that the number of layer in building tree is not too high, according to the sample size, $10 \%-20 \%$ of the sample is suggested. Second is the maximum allowable drop in space of the leaf node (MaxDrop). In the process of building tree, it usually sets the maximum allowable sample in the leaf node as first priority, and the MaxDrop needs to deploy precisely by examining and debugging in the detailed drawing of the tree. Considering Partial Least Squares method is applied to the leaf nodes and it can deal with multi-independent and multi-dependent variable problem, PMT can be stretched directly as a new way of supporting multivariate data analysis with multi-independent and multi-dependent variable. When dividing the origin sample data set, not only should the total variance of multidependent variable take into account about the entirety, but the mixed total variance produced after dividing the tree should also be considered about the entirety. The detailed flow chart of specific algorithm of PMT is shown in Figure I. Based on the principle of maximum fluctuation drop in space of the total variance in Model Tree [9], PMT looks for the division attribute and chooses the attribute with the maximum reduction of the expected error as the best division attribute in the current node. To measure the consistence of numeric data, it sets the sum of squares of subtraction between the average and the independent variable of every data as measuring rule, namely, the reduction of the total variance, the formula is as follows: 


$$
S D R=s d(T)-\sum_{i=1}^{2} \frac{\left|T_{i}\right|}{T} \llbracket s d\left(T_{i}\right)
$$

Here, $T_{1}$ and $T_{2}$ is the data subset of two child nodes, and they are divided from parent node $T$ by division attribute.

For the number of the principal component in PLSR, it will not extract all the principal component to build the model. The existing literature already shows [10] that the number of the principal component is $m$ when extracted information can represents the most of the origin sample data or there is little disturbance residual information, it can stop extracting, particularly, the two is equal to each other. Therefore, normally, in the analysis of Partial Least Squares, when it is up to $80 \%$ for the percentage rate of the extracting information to stop extract information from the origin regression data set. In the later calculation, it applies this rule uniformly to judge the extraction number of the principle component.

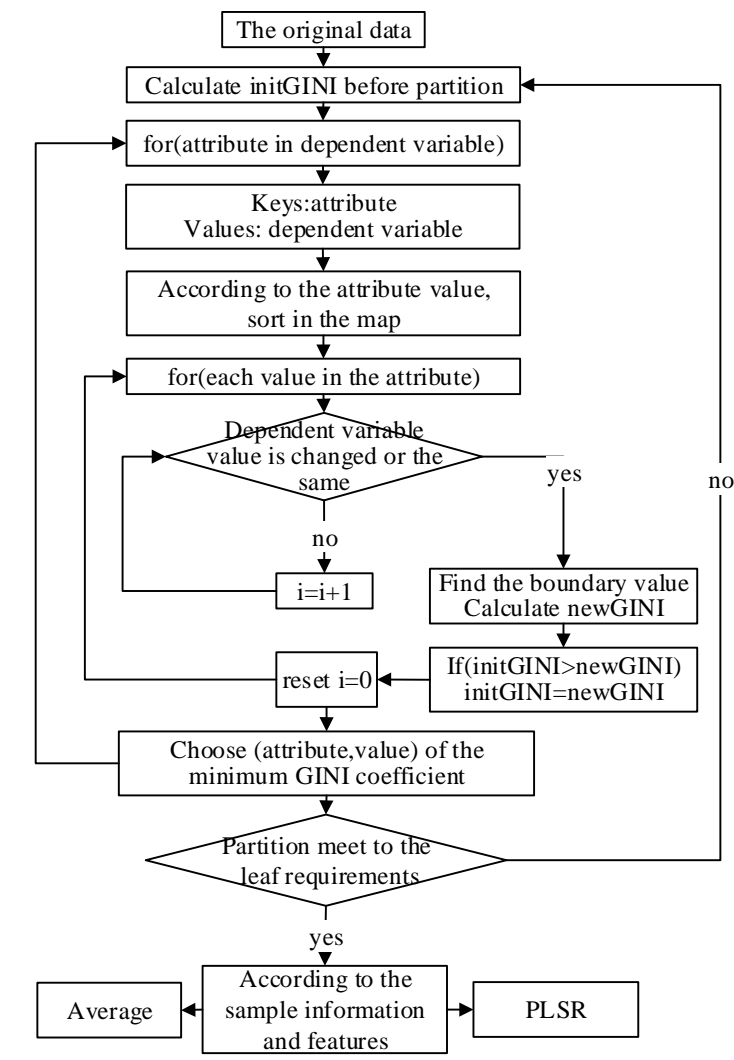

FIGURE I. THE ALGORITHM FLOW GRAPH OF PARTIAL LEAST SQUARES MODEL TREE.

\section{RANDOM FOREST OF PARTIAL LEAST SQUARES}

One branch of Random Forest is numerical regression, called Random Forest Regression algorithm. Random Forest Regression is an ensemble learning method to solve the same problem by lots of distinct versions of the individual learner, thus heavily improving the learning ability and generalization of system [11]. Because PMT is a weak individual learner like regression tree, it has a power of integrated into ensemble learning and enhances the ability of generalization for the unknown data.

Definition 2: Using the bootstrap random sampling on the sample data, called as the training set in the bag (ITB), building PMT model on many distinct versions of the training set, the predicted result of the final model is made decisions by the mean in many PMTs. This model is called as Random Forest Regression of Partial Least Squares (PLS-RFR).

Given the basic regression tree is bigger, complex and difficult to explain, in PLS-RFR, the basic regression tree isn't as individual learner any longer but PMT instead. The main idea is as followed: first, adopting the Bootstrap sampling to copy the learning training set, generating lots of distinct versions of the sub-learning training set; then, building lots of distinct versions of the sub-learner to go along with the sublearning training set, namely, Partial Least Squares Model Tree; last, deciding the final predicted result by the mean. Because of the weak learning ability of PMT, the ensemble learning can enhance the generalization of PLS-RFR. Hence, PMT is a kind of localized nonlinear modeling method for complex data. The main algorithm is as follows:

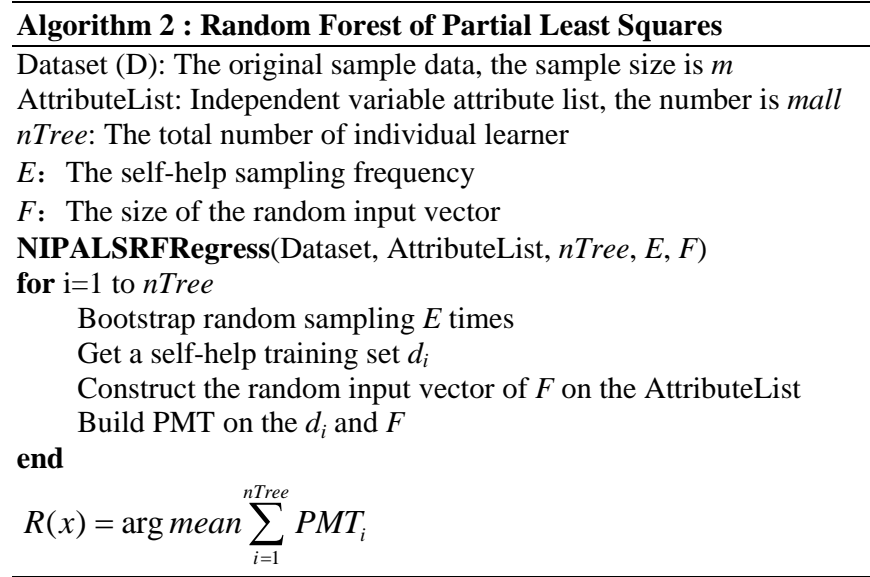

The algorithm 2 get improved based on PMT and RFR. Considering the features of RFR, besides the two parameters of PMT, there are another three parameters involved and parameters recommendation is the same as the traditional RFR [12]: one is the random input vector $F, F$ generally sets to $\sqrt{q}$ or $\operatorname{int}\left(1+\log _{2} M\right)$, here, int represents the rounded down among them. Second is the sampling frequency of Bootstrap $E$, it is generally recommended as the capacity of origin sample data set $m$. Third, it is the number of individual learner ntree, which is decided by the change of residual by increasing the number of the random tree. With the increasing of the size of the tree, the residual will be stable. At the beginning, ntree sets 100 in the training process of modeling. The three parameters still take example by the recommended value of RFR. Since the PLS-RFR is based on the PMT, after stretching PMT to analyze the data with multi-independent and multi-dependent variable, PLS-RFR also supports multiindependent and multi-dependent variable analysis. The flow graph of PLS-RFR is as followed: 


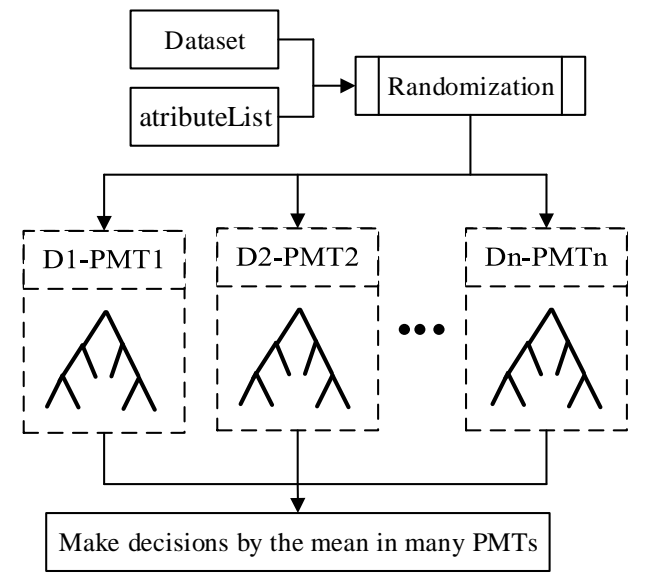

FIGURE II. THE ALGORITHM FLOW GRAPH ABOUT PARTIAL LEAST SQUARES OF RANDOM FORESTS REGRESSION

In Figure II, after constructing ITB and the random input vector on the original sample data by using the bootstrap random sampling, lots of distinct versions of PMT are built, the final predicted result is decided by the average.

\section{EXPERIMENTAL ANALYSIS}

To observe the regression model effect about PMT and PLS-RFR, it adopt the traditional Multiple Regression (MR), the traditional PLSR and the traditional Random Forests Regression (RFR) as a contrast. Based on this, it divides the original data randomly into the proportion of $7: 3$, the part of $70 \%$ is the training set to build the model, and the remaining is the testing set to validate the model. There are five indicators for investigation, namely, Sum of Squares for Error of Train (SSETrain), Sum of Squares for Error of Test (SSETest), coefficient of determination (R-Square) and time consuming (Time, Unit: ms). In order to facilitate the experiment, verifying the effect of the model and establishing enough testing set for contrast, it defines that the original sample data obtained here is greater than or close to 50 . Here, it adopts the experimental data of MaXingShiGan decoction of the monarch drug to treat the asthma or cough and five sample sets in the UCI Machine Learning Repository [13] to compare.

\section{A. The Experiment of MaXingShiGan Decoction of the Monarch Drug to Treat the Asthma}

TABLE I shows the part of experiment data of MaXingShiGan decoction of the monarch drug to treat the asthma for rats in the key laboratory of Modern Preparation of TCM, Ministry of Education in Jiangxi University of Traditional Chinese medicine, a total of 46 samples, it is about the impact of pharmacological indicators about the blood medicine composition in rats under 10 distinct dosage of herbal ephedra respectively. There are five compositions about the blood medicine composition in rats, namely, ephedrine, pseudoephedrine, methyl ephedrine, wild black cherry glycosides and licorice glycosides. There are two pharmacological indicators namely, incubation period (Unit: s) and cough duration (Unit: min). Based on this, there are five independent variables and two dependent variables, it divides the original data randomly into the proportion of $7: 3$, the part of $70 \%$ is the training set to build the model, and the remaining is the testing set to validate the model. Then, it compares the model result between PMT, PLS, RFR, MR and PLS-RFR by JAVA programming. The experiment results are shown in TABLE II.

TABLE I. THE EXPERIMENTAL DATA OF THE MAXINGSHIGAN DECOCTION OF THE MONARCH DRUG ABOUT THE TREATMENT OF THE ASTHMA CHANGING THE EPHEDRA DOSE

\begin{tabular}{ccccccc}
\hline $\begin{array}{c}\text { ephe- } \\
\text { drine }\end{array}$ & $\begin{array}{c}\text { pseudo- } \\
\text { phedrine }\end{array}$ & $\begin{array}{c}\text { methyl } \\
\text { ephedrine }\end{array}$ & $\begin{array}{c}\text { wild } \\
\text { black } \\
\text { cherry } \\
\text { glycol- } \\
\text { sides }\end{array}$ & $\begin{array}{c}\text { licorice } \\
\text { glycol- } \\
\text { sides }\end{array}$ & $\begin{array}{c}\text { incubation } \\
\text { period } \\
\text { (Unit: s) }\end{array}$ & $\begin{array}{c}\text { cough } \\
\text { duration } \\
\text { (Unit: } \\
\text { min) }\end{array}$ \\
\hline 1.04 & 0.47 & 0.19 & 0 & 0.47 & 68 & 28 \\
0.95 & 0.53 & 0.17 & 1.67 & 0.48 & 44 & 22 \\
1.99 & 1.92 & 0.17 & 0 & 0.18 & 108 & 10 \\
1.09 & 0.43 & 0.41 & 0 & 0.42 & 71 & 19 \\
2.31 & 1.89 & 0.08 & 0.121 & 0.21 & 150 & 9 \\
2.96 & 2.96 & 0.353 & 0.216 & 0.83 & 49 & 24 \\
1.11 & 0.89 & 0.92 & 0 & 0.87 & 54 & 9 \\
3.41 & 2.85 & 0.293 & 0 & 0.9 & 80 & 7 \\
5.59 & 4.92 & 0.4363 & 0 & 0.89 & 52 & 17 \\
5.08 & 5.22 & 0.4063 & 0.977 & 0.88 & 80 & 12 \\
$\ldots \ldots$ & $\ldots \ldots$ & $\ldots \ldots$ & $\ldots \ldots$ & $\ldots \ldots$ & $\ldots \ldots$ & $\ldots \ldots$ \\
\hline
\end{tabular}

TABLE II. COMPARISON OF FIVE METHODS IN THE EXPERIMENTAL DATA OF THE MAXINGSHIGAN DECOCTION OF THE MONARCH DRUG ABOUT THE TREATMENT OF THE ASTHMA

\begin{tabular}{ccccc}
\hline & Time & R-Square & SSETrain & SSETest \\
\hline MR & 2 & 0.2198 & 20193.6746 & 9848.1760 \\
PLSR & 32 & 0.1483 & 20537.1629 & 9910.0989 \\
PMT & 8 & 0.4262 & 14851.2187 & 9535.6027 \\
RFR & 140 & 0.3474 & 16892.1152 & 8921.9143 \\
RFR-PLS & 142 & 0.5159 & 12529.7715 & 8714.3140
\end{tabular}

In TABLE II, for the Maxingshigan Decoction of the monarch drug about the treatment of the asthma of 2 independent variables, PLSR don't show a good expected effect, the explanation level reached only $14.83 \%$, RFR is only $34.74 \%$, and PMT is $42.62 \%$. However, the improved PLSRFR is up to $51.59 \%$ in the ensemble learning, Sum of Squares for Error has a certain degree of decline in the training set and testing set.

\section{B. The Experiment of MaXingShiGan Decoction of the Monarch Drug to Treat the Cough}

TABLE III shows the part of experiment data of MaXingShiGan decoction of the monarch drug to treat the cough for rats in the key laboratory of Modern Preparation of TCM, Ministry of Education in Jiangxi University of Traditional Chinese Medicine, a total of 63 sample data, it is about the impact of pharmacological indicators about the blood medicine composition in rats under 10 distinct dosage of almonds respectively. There are five compositions about the blood medicine composition in rats, namely, ephedrine, pseudoephedrine, methyl ephedrine, wild black cherry glycosides and laetrile. There are one pharmacological 
indicators namely, cough times (Unit: times). Based on this, there are five independent variables and one dependent variables, it divides the original data randomly into the proportion of $7: 3$, the part of $70 \%$ is the training set to build the model, and the remaining is the testing set to validate the model. Then, it compares the model result between PMT, PLS, RFR, MR and PLS-RFR by JAVA programming. The experiment results are shown in TABLE IV.

TABLE III THE EXPERIMENTAL DATA OF THE MAXINGSHIGAN DECOCTION OF THE MONARCH DRUG ABOUT THE TREATMENT OF THE COUGH CHANGING THE ALMOND DOSE

\begin{tabular}{|c|c|c|c|c|c|}
\hline ephedrine & $\begin{array}{l}\text { pseudo- } \\
\text { ephedrine }\end{array}$ & $\begin{array}{c}\text { methyl } \\
\text { ephedrine }\end{array}$ & laetrile & $\begin{array}{l}\text { wild black } \\
\text { cherry } \\
\text { glycosides }\end{array}$ & $\begin{array}{l}\text { cough } \\
\text { times } \\
\text { (Unit: } \\
\text { times) }\end{array}$ \\
\hline 3.74 & 3.78 & 0.52 & 33.2 & 24.4 & 41 \\
\hline 373.59 & 217.94 & 16.38 & 0.629 & 2.24 & 58 \\
\hline 402 & 369.93 & 48.46 & 0.785 & 1.87 & 25 \\
\hline 9 & 9.35 & 0.54 & 16.6 & 16.6 & 39 \\
\hline 18.3083 & 21.5 & 1.08 & 4.81 & 40.1 & 22 \\
\hline 42.532 & 42.6 & 2.8383 & 2.3 & 8.32 & 47 \\
\hline 2.25 & 2.975 & 0.493 & 40 & 40 & 42 \\
\hline 20.1733 & 19.95 & 0.98 & 4.77 & 10.2 & 23 \\
\hline 43.296 & 37.6 & 2.5483 & 2.852 & 9.45 & 45 \\
\hline 21.7417 & 24.36 & 1.17 & 4.62 & 26 & 38 \\
\hline$\ldots \ldots$ & $\ldots \ldots$ & $\ldots \ldots$ & $\ldots \ldots$ & $\ldots \ldots$ & $\ldots \ldots$ \\
\hline
\end{tabular}

TABLE IV. THE RESULT COMPARISON OF FIVE METHODS IN THE EXPERIMENTAL DATA OF THE MAXINGSHIGAN DECOCTION OF THE MONARCH DRUG ABOUT THE TREATMENT OF THE COUGH

\begin{tabular}{ccccc}
\hline & Time & R-Square & SSETrain & SSETest \\
\hline MR & 2 & 0.1203 & 3468.3287 & 1622.4532 \\
PLSR & 31 & 0.0587 & 3710.8791 & 1533.7792 \\
PMT & 16 & 0.6671 & 1312.3766 & 1524.6328 \\
RFR & 109 & 0.0975 & 3558.0023 & 1777.0677 \\
$\begin{array}{c}\text { RFR- } \\
\text { PLS }\end{array}$ & 124 & 0.3433 & 2588.8972 & 1553.9246 \\
\hline
\end{tabular}

In TABLE IV, for the Maxingshigan Decoction of the monarch drug about the treatment of the cough of 1 independent variables, PMT and PLS-RFR is nearly up to $66.71 \%$ and $34.33 \%$ in the explanation level. PMT drop to 1312.3766 and 1524.6328 about Sum of Squares for Error on the training set and testing set. Simultaneously, PLS-RFR falls to 2588.8972 and 1553.9246.

\section{UCI Machine Learning Repository}

To validate the feasibility and effectiveness of PMT and PLSR, it adopts five sample sets in the UCI Machine Learning Repository. TABLE V shows the basic information about five machine learning data set.

TABLE V. THE SAMPLE SETS IN THE UCI MACHINE LEARNING REPOSITORY

\begin{tabular}{cccccc}
\hline Dataset & Abbreviation & $\begin{array}{c}\text { The number of } \\
\text { independent variable }\end{array}$ & $\begin{array}{c}\text { The number of } \\
\text { dependent variable }\end{array}$ & The training & The testing \\
set & \multicolumn{2}{c}{ set } \\
\hline Concrete Compressive & Concrete & 8 & 1 & 721 & 309 \\
Strength & Slump & 7 & 3 & 72 & 31 \\
Slump & Yacht & 6 & 1 & 216 & 92 \\
Yacht & Housing & 13 & 1 & 354 & 152 \\
Hydrodynamics & Airfoil & 5 & 1 & 1052 & 451 \\
Housing & Airfoil & & & & \\
Self-Noise & & & & & \\
\hline
\end{tabular}

In the process of experiment, making the model to be optimal by adjusting the parameter of model, it compares the model result under the condition of same parameters. First, it divides the original data randomly into the proportion of $7: 3$, the part of $70 \%$ is the training set to build the model, and the remaining is the testing set to validate the model. Then, it compares the quality and effect under the same learning training set in several ways. Last, it uses JAVA to accomplish this process. The experimental results are shown in TABLE VI-X.

TABLE VI. THE RESULT COMPARISON OF FIVE METHODS IN THE CONCRETE COMPRESSIVE STRENGTH DATASET

\begin{tabular}{ccccc}
\hline & Time & R-Square & SSETrain & SSETest \\
\hline MR & 7 & 0.5824 & 78433.8274 & 33045.0562 \\
PLSR & 50 & 0.5739 & 80026.3124 & 33655.5497 \\
PMT & 281 & 0.905 & 17835.0102 & 14541.2386 \\
RFR & 338 & 0.3478 & 122501.7651 & 64488.188 \\
RFR-PLS & 385 & 0.7496 & 47029.7116 & 20739.7566 \\
\hline
\end{tabular}

TABLE VII. THE RESULT COMPARISON OF FIVE METHODS IN THE SLUMP DATASET

\begin{tabular}{ccccc}
\hline & Time & R-Square & SSETrain & SSETest \\
\hline MR & 2 & 0.4656 & 15432.9726 & 7212.6384 \\
PLSR & 47 & 0.5891 & 15594.1733 & 7729.8852 \\
PMT & 15 & 0.7925 & 6918.391 & 7554.5867 \\
RFR & 171 & 0.285 & 20649.4744 & 11250.2986 \\
RFR-PLS & 344 & 0.6173 & 11052.1156 & 6536.1835 \\
\hline
\end{tabular}

TABLE VIII. THE RESULT COMPARISON OF FIVE METHODS IN THE YACHT HYDRODYNAMICS DATASET

\begin{tabular}{ccccc}
\hline & Time & R-Square & SSETrain & SSETest \\
MR & 2 & 0.6587 & 16076.1336 & 8159.7909 \\
PLSR & 41 & 0.6586 & 16076.4914 & 8151.2763 \\
PMT & 14 & 0.9967 & 155.7321 & 266.439 \\
RFR & 107 & 0.8151 & 8706.8571 & 4900.1754 \\
RFR-PLS & 168 & 0.987 & 614.3444 & 560.7723 \\
\hline
\end{tabular}


TABLE IX. THE RESULT COMPARISON OF FIVE METHODS IN THE HOUSING DATASET

\begin{tabular}{ccccc}
\hline & Time & R-Square & SSETrain & SSETest \\
\hline MR & 1 & 0.8733 & 3184.3654 & 83131.7422 \\
PLSR & 48 & 0.8733 & 3184.4137 & 81262.7214 \\
PMT & 198 & 0.9469 & 1333.597 & 70160.8003 \\
RFR & 439 & 0.6859 & 7892.3088 & 15598.435 \\
RFR-PLS & 550 & 0.9115 & 2224.4969 & 8247.0806 \\
\hline
\end{tabular}

TABLE X. THE RESULT COMPARISON OF FIVE METHODS IN THE AIRFOIL SELF-NOISE DATASET

\begin{tabular}{ccccc}
\hline & Time & R-Square & SSETrain & SSETest \\
\hline MR & 2 & 0.5026 & 24374.287 & 10311.6879 \\
PLSR & 50 & 0.5024 & 24381.2663 & 10289.1888 \\
PMT & 108 & 0.8979 & 5003.6441 & 3877.8539 \\
RFR & 283 & 0.2014 & 39131.9915 & 18286.096 \\
RFR-PLS & 249 & 0.6429 & 17497.5751 & 7568.6188 \\
\hline
\end{tabular}

In TABLE VI-X. it will have a good visual effect shown by chart. For MR, PLSR and RFR, the explanation level gets improved about five sample sets of the UCI Machine Learning Repository by PMT and PLS-RFR. Simultaneously, SSETrain and SSETest have a certain degree of decline. PLS-RFR gets obviously than PMT about the generalization. It shows that PMT and PLS-RFR have a certain degree of validity and feasibility.

\section{CONCLUDING REMARKS}

Through the above analysis, we can obtain the following conclusions:

Firstly, For the problem that Partial Least Squares Regression is lack of theoretical guidance of rules by the quasi linearization rule to achieve the nonlinear, and the accuracy declines in face of the unknown variables distribution, and the information is easy to loss about the mean treatment of the leaf in the Regression Tree and the Random Forest Regression, by adopting the PLSR at the leaf nodes, the information loss issue gets avoided when the leaf nodes is directly for mean with appropriate samples. Simultaneously, by applying the dividable principle of the attribute in Regression Tree, it makes several linear segments of Partial Least Squares approach unknown variable distribution to achieve the nonlinear regression.

Secondly, by Bootstrap sampling the origin data set, choosing randomly features in all attributes by the number, constructing various copies of the training dataset of distinct size, building distinct versions of PMT for ensemble learning, PLS-RFR is constructed, enhancing the generalization of PMT.

Thirdly, for the experiment data of Maxingshigan Decoction of the monarch drug about the treatment of the asthma or cough and five sample sets in the UCI Machine Learning Repository, the explanation level get increased after adopting the improved model, it shows that PMT and PLSRFR have a certain degree of validity and feasibility.
Fourthly, it is able to pull PLS, PMT, PLS-RFR into the data of Traditional Chinese Medicine to provide better technical support.

\section{ACKNOWLEDGMENT}

This work is supported by the Key Laboratory of modern preparation of Traditional Chinese Medicine (TCM), Ministry of education and two national natural science foundations (61363042 \& 61562045). This research also is supported by a major project of Jiangxi Natural Science Foundation (20152ACB20007).

\section{REFERENCES}

[1] Z. Q. Jia, J. Y. Cai, Y. Y. Liu. Real-time performance reliability evaluation method of small-sample based on improved Boostrap and Bayesian Bootstrap. Journal of Computer Applications. Application Research of Computers. vol. 26, no. 08, pp. 2851-2854, 2009.

[2] J. R. Quinlan. Learning with continuous classes. Proceedings of the 5th Australian joint Conference on Artificial Intelligence. Singapore, 1992.

[3] H. J. Li, Z. X. Wang, L. M. Wang, et al. Bayesian-network based Regression Tree Learning Algorithm. Chinese Journal of Scientific Instrument. vol. z3, pp. 387-388, 2004.

[4] K. Zhang, Z. C. Mu, X. H. Chang, et al. On A New Model Tree Algorithm and Its Application . Control Engineering of China. vol. 15, no. 01, pp. 103-106, 2008.

[5] H. Abdi, L Williams. Partial Least Squares Methods: Partial Least Squares Correlation and Partial Least Square Regression. Reisfeld B, Mayeno A N, Humana Press, pp. 930, 549-579, 2013.

[6] H. W. Wang, Z. B. Wu, H. Meng, et al. Linear and nonlinear method of partial least squares regression. Beijing: National Defend In-dustry Press, 2006

[7] Y. Liu. Response Surface Modeling by Local Kernel Partial Least Squares. Tsinghua University, 2013.

[8] Z. L. Hao, J. Q. Du, G. L. Wang, et al. Analysis of partial least squares method combining with SBM. Computer engineering and design. pp. 2896-2900, 2014.

[9] F. H. Shang, W. Zhang. Study on inferring interwell connectivity of injection-production system based on decision tree. Application Research of Computers. vol. 30, no. 07, pp. 2051-2054, 2013.

[10] J. X. Guo. Study on Improved High-Dimension and Nonlinear Partial Least-Squares Regression Method and Applications. Tianjin University, 2010.

[11] W. X. Xi, X. Y. Tang. Pedestrian detection based on random forest and support vector machine. Journal of Computer Applications. vol. 34, no. S2, pp. 283-285, 2014.

[12] Z. F. Cao. Study on optimization of random forests algorithm. Capital University of Economics and Business, 2014.

[13] UCI machine learning repository. 2014. 